\title{
The effects of circularly polarized light on amino acid enantiomers produced by the UV irradiation of interstellar ice analogs
}

\author{
M. Nuevo ${ }^{1,2}$, U. J. Meierhenrich ${ }^{3,4}$, G. M. Muñoz Caro ${ }^{1,5}$, E. Dartois ${ }^{1}$, L. d'Hendecourt ${ }^{1}$,
} D. Deboffle ${ }^{1}$, G. Auger ${ }^{6}$, D. Blanot ${ }^{6}$, J.-H. Bredehöft ${ }^{3}$, and L. Nahon ${ }^{7,8,9}$

\author{
1 Institut d'Astrophysique Spatiale (IAS), Bât. 121, Université Paris-Sud, 91405 Orsay Cedex, France \\ 2 Graduate Institute of Astronomy, National Central University, No. 300, Jhongda Rd, Jhongli City, Taoyuan County 32049, Taiwan \\ e-mail: mnuevo@astro.ncu.edu.tw \\ 3 Universität Bremen, Physikalische Chemie NW2, FB02, Leobener Str., 28359 Bremen, Germany \\ ${ }^{4}$ Laboratoire de Chimie Bioorganique, UMR 6001 CNRS, Université de Nice-Sophia Antipolis, Faculté des Sciences, Parc Valrose, \\ 06108 Nice Cedex 2, France \\ 5 Centro de Astrobiología, INTA-CSIC, carretera de Ajalvir, km 4, Torrejón de Ardoz, 28850 Madrid, Spain \\ 6 Institut de Biochimie et de Biophysique Moléculaire et Cellulaire, Bât. 430, Université Paris-Sud, 91405 Orsay Cedex, France \\ 7 Laboratoire pour l'Utilisation du Rayonnement Electromagnétique (LURE), Bât. 209D, Université Paris-Sud, 91405 Orsay Cedex, \\ France \\ 8 CEA/DRECAM/SPAM and LFP/CNRS URA 2453, Bât. 522, CE de Saclay, 91191 Gif-sur-Yvette Cedex, France \\ 9 SOLEIL, L'Orme des Merisiers, St Aubin, BP 48, 91192 Gif-sur-Yvette Cedex, France
}

Received 15 September 2004 / Accepted 24 May 2006

\section{ABSTRACT}

\begin{abstract}
Aims. Two irradiation experiments on interstellar ice analogs at $80 \mathrm{~K}$ under interstellar-like conditions were performed with the LURE SU5 synchrotron beamline to assess, for the first time, the photochemical effect of circularly polarized ultraviolet light (UV CPL) at $167 \mathrm{~nm}(7.45 \mathrm{eV})$ with right and left polarizations on such ice mixtures.

Methods. This effect was measured by determining the enantiomeric excesses (e.e.s) for two amino acids formed in the solid organic residues produced during the subsequent warm-up of the irradiated samples to room temperature: alanine, the most abundant chiral proteinaceous amino acid produced (both polarizations) and 2,3-diaminopropanoic acid (DAP), a non-proteinaceous amino acid (rightpolarization experiment). These excesses were compared to those measured for the same amino acids produced after unpolarized UV irradiation of the same ice mixtures (expected to be zero), in order to determine the contribution of CPL only. A careful estimate of all the associated uncertainties (statistical and systematic errors) was also developed.

Results. It appears that the enantiomeric photochemical effect at this wavelength is weak, since both alanine and DAP e.e.s were found to be small, at most of the order of $1 \%$ in absolute values, and tends to be inconclusive since the effects obtained for both amino acids and both polarizations are not those expected. In light of these results, the hypothesis that CPL may be one source responsible for the e.e.s measured for such amino acids in some meteorites and, more generally, that CPL may be directly related to the origin of biomolecular homochirality on Earth is discussed.
\end{abstract}

Key words. molecular processes - polarization - methods: data analysis - methods: laboratory - ISM: molecules - infrared: ISM

\section{Introduction}

Experiments simulating ultraviolet (UV) photo-processing of icy grain mantles in the interstellar or circumstellar media (ISM or CSM, respectively), and subsequent warm-up to room temperature are known to produce organic residues made of a large variety of complex organic molecules (Greenberg \& Yencha 1973; Hagen et al. 1979; Agarwal et al. 1985; d'Hendecourt et al. 1985; Briggs et al. 1992; Bernstein et al. 1995; Muñoz Caro \& Schutte 2003), including amino acids (Muñoz Caro et al. 2002; Bernstein et al. 2002; Nuevo et al. 2005).

Most of these amino acids are chiral, i.e. they exist in two forms called levorotatory (L) and dextrorotatory (D) enantiomers which are non-superimposable mirror image of one another. These adjectives refer to the fact that chiral molecules have the property to make the polarization plane of a linearly polarized light rotate when passing through a chiral solution. The rotation angle induced by one enantiomer will be opposite to the angle induced by the other. A racemic mixture, i.e. a mixture containing as much L-enantiomers as D-enantiomers, will not display such an effect.

Because of their specific interaction with light, chiral molecules were early assumed to be affected by circularly polarized light (CPL), e.g. they could be photo-produced or photo-destroyed by a selective interaction with one enantiomer. Experiments performed with circurlarly and elliptically polarized light (CPL and EPL, respectively) on racemic leucine in solution at $212.8 \mathrm{~nm}$ showed that polarized light induces asymmetric photolysis. Enantiomeric excesses (e.e.s) from 1.98 to 4.68\% with CPL (Flores et al. 1977; Bonner \& Bean 2000), and from 2.56 to $3.88 \%$ with EPL (Bonner \& Bean 2000), were induced by photo-decomposition of racemic leucine.

Life uses only L enantiomers of amino acids to form proteins, and D enantiomers of riboses for DNA and RNA. The origin of this property, called biomolecular homochirality, is still a mystery and several mechanisms likely to create enantiomeric 
excesses in the ISM were proposed to explain its origin (see Jorissen \& Cerf 2002 and references therein) and make the link with the excesses found for some amino acids in meteorites such as Murchison (Engel \& Macko 1997; Cronin \& Pizzarello 1997, 1999; Pizzarello et al. 2003) and Murray (Cronin \& Pizzarello 1999).

Among the proposed mechanisms, the irradiation of icy mantles on the surface of dust grains by UV CPL, in the presolar cloud or the proto-planetary nebula from which our Solar System formed, is a potential candidate (Rubenstein et al. 1983). To check this hypothesis one must be able to assess the effect(s) of CPL on the complex chiral molecules produced, and find an astrophysical source providing such radiation in space. CPL could for instance be provided either by cyclotron radiation emitted by binary systems containing an accreting magnetic white dwarf (polars) or an isolated magnetic white dwarf with high magnetic fields $\left(10^{7}\right.$ to $\left.10^{8} \mathrm{G}\right)$ (Bailey 2001). Scattering of unpolarized light by magnetically aligned spheroidal interstellar grains in reflection nebulae (Bailey 2001) was proposed to explain the emission of the circularly polarized $(17 \%)$ infrared radiation observed in OMC-1, a molecular cloud in Orion (Bailey et al. 1998), where UV light could also be circularly polarized with a lower polarization rate (Buschermöhle et al. 2005).

In this paper we report the photo-processing of interstellar ice analogs irradiated with synchrotron UV CPL at LURE, the French synchrotron radiation center in Orsay, at $167 \mathrm{~nm}$ $(7.45 \mathrm{eV})$ with right and left polarizations in two independent experiments, named L1 and L2, respectively. We identified eight amino acids in the residues produced, and determined the L-enantiomeric excesses (arbitrary choice) for two chiral amino acids, namely alanine (Ala, proteinaceous) and 2,3-diaminopropanoic acid (DAP, non-proteinaceous, recently identified in the Murchison meteorite by Meierhenrich et al. in 2004). For these two compounds the enantiomeric excesses could be determined in the first experiment (L1, right polarization). In the second experiment (L2, left polarization), only the alanine excess could be determined, because of a lower signalto-signal ratio in the DAP chromatogram. We then compare the results obtained for the same ice mixtures irradiated by unpolarized UV light in order to quantify the real contribution of CPL on the produced amino acids. Several sources of errors associated with the determination of these excesses are also clearly identified and tentatively estimated in detail for the first time in such experiments. Finally, the derived excesses are compared to those measured for amino acids in Murchison and Murray meteorites in order to check whether CPL may be responsible for the excesses observed in extraterrestrial matter such as carbonaceous chondrites.

\section{Experiments}

\subsection{UV irradiation of ices at $80 \mathrm{~K}$}

In a high vacuum chamber evacuated by a turbo-molecular pump (Leybold Turbovac 361) to a pressure of a few $10^{-7} \mathrm{mbar}$, gas mixtures were deposited onto an infrared (IR) transparent $\mathrm{MgF}_{2}$ window fixed on a cold finger and cooled down to $80 \mathrm{~K}$. This temperature was chosen since the residues produced after irradiation of ices at $\sim 12$ and $80 \mathrm{~K}$ appear to be similar (Muñoz Caro \& Schutte 2003). The gas mixtures were previously prepared in a stainless steel gas line also evacuated by a turbo-molecular pump (Alcatel ATP 150, vacuum pressure: $\sim 10^{-5}$ mbar). The deposited ices were then irradiated with
UV photons. Two types of irradiation were performed: with the SU5 undulator-based high resolution variable polarization VUV synchrotron beamline at LURE (Nahon et al. 2001) at $167 \mathrm{~nm}$ (7.45 eV), and with a microwave-powered (Opthos) $\mathrm{H}_{2}$ flow discharge UV lamp at the IAS, providing mainly $\sim 160 \mathrm{~nm}$ $(7.75 \mathrm{eV})$ and Lyman- $\alpha$ (121.6 nm, i.e. $10.25 \mathrm{eV})$ unpolarized photons.

Both irradiated gas mixtures had a $\mathrm{H}_{2} \mathrm{O}:{ }^{13} \mathrm{CH}_{3} \mathrm{OH}: \mathrm{NH}_{3}=$ 1:1:1 composition. $\mathrm{H}_{2} \mathrm{O}$ was purified to a $18.2 \mathrm{M} \Omega \mathrm{cm}$ resistivity (Millipore Direct-Q 5), ${ }^{13} \mathrm{CH}_{3} \mathrm{OH}$ was from Sigma $\left(99 \%{ }^{13} \mathrm{C}\right)$ and $\mathrm{NH}_{3}$ from Messer $(99.98 \%$ purity). This mixture, consisting of compounds observed in dense molecular cloud ices (e.g. Dartois et al. 2002; Gibb et al. 2004), was chosen after analysing several residues produced from different mixtures under different experimental conditions (temperature, irradiation time) with a Hitachi L-8800 Amino Acid Analyzer equipped with a non-chiral liquid chromatography column (2620 MSCPS type, length: $80 \mathrm{~mm}$, inner diameter: $4.6 \mathrm{~mm}$ ) and a postcolumn ninhydrin-reaction device. Those analyses allowed us to select the best experimental parameters and starting gas mixture leading to the production of sufficient amounts of amino acids for quantification. Typically, the total abundances found for the amino acids produced in our residues range from 10 to $60 \mathrm{nmol}$ for each organic residue, with individual amounts ranging from 1 to $29 \mathrm{nmol}(0.1$ to $2.2 \mu \mathrm{g})$ for the most abundant amino acids, namely glycine and alanine.

The relative proportions of the gases were controlled by their partial pressures. Methanol was ${ }^{13} \mathrm{C}$-labelled in order to analyse the contribution of the amino acids produced in the irradiated ice mixtures, thus avoiding confusion with any biological or pump oil contaminants. $\mathrm{MgF}_{2}$ windows with no deposition were irradiated with the same $\mathrm{H}_{2}$ lamp at the IAS under similar conditions and showed no organic contaminant (see Fig. 7, (c) chromatograms). The IR spectra at $80 \mathrm{~K}$ of the ice mixtures irradiated in the L1 and L2 (LURE) experiments are given in Fig. 1 and show strong similarities between the two mixtures.

For the CPL irradiation, the experimental setup was connected to the SU5 synchrotron beamline, $1.35 \mathrm{~m}$ donwstream of the focal point. The irradiation was performed using the white beam provided by the OPHELIE variable polarization undulator (Nahon et al. 1997) feeding the SU5 beamline with its grating set at the 0th order, taking advantage of its tunable central peak energy. Simultaneous deposition/irradiation was performed, with right- (RCPL, 43-h irradiation, L1 sample) and left-circularly polarized light (LCPL, 56 h, L2 sample), within a 3 month interval. The photons were circularly polarized with measured absolute polarization rates of $91 \%$ and $94 \%$ for RCPL and LCPL, respectively, in the 7-8 eV range (Nahon \& Alcaraz 2004). The integrated photon flux for the whole beam was close to $10^{14}$ photons $\mathrm{s}^{-1}$, of which more than $70 \%$ of the photons had energies within the full width half maximum of the flux density curve (Fig. 2b), comparable with the photon flux at $160 \mathrm{~nm}$ and above provided by $\mathrm{H}_{2}$ discharge UV lamps. The calculated gas deposition flows for the mixtures within the photolysed area were $1.0 \times 10^{13}$ and $2.0 \times 10^{13}$ molec $\mathrm{cm}^{-2} \mathrm{~s}^{-1}$, implying a number of photons per deposited molecule of 6.8 and 3.6, for L1 and L2 experiments, respectively. However, the SU5 footprint had a size less than $25 \%$ of the deposited ices that were actually irradiated during CPL experiments (L1 and L2). The unphotolysed fraction of the ices evaporated during the warm-up. Thus, when removing the samples from the cryostat at room temperature, the residues on the windows had the same size and shape as the SU5 footprint, indicating that they were produced by CPL only. 


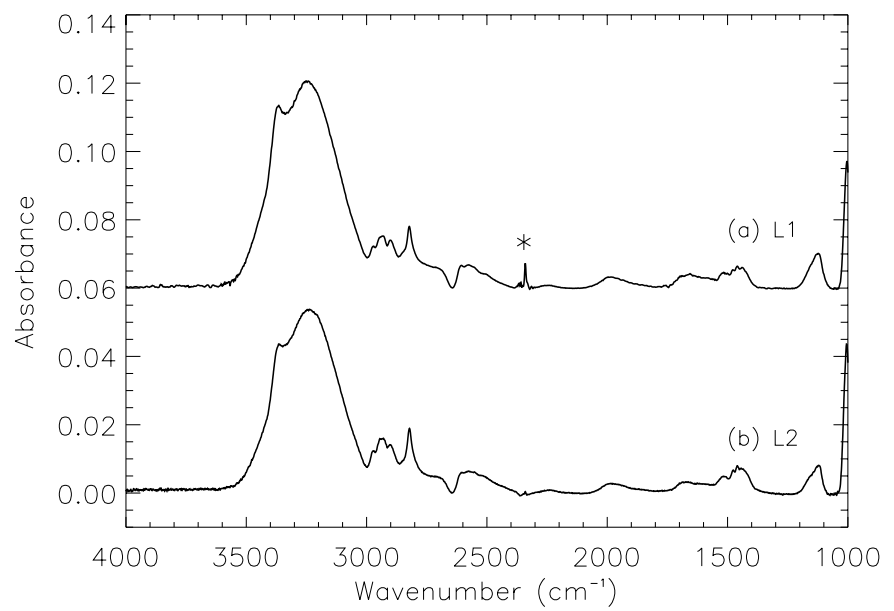

Fig. 1. Infrared spectra of the $\mathrm{H}_{2} \mathrm{O}:{ }^{13} \mathrm{CH}_{3} \mathrm{OH}: \mathrm{NH}_{3}=1: 1: 1$ ice mixtures irradiated in a) $\mathrm{L} 1$ and b) $\mathrm{L} 2$ experiments at $80 \mathrm{~K}$, with RCPL and LCPL, respectively, where the spectrum of L1 (a) is offset for clarity. The spectra show the same features over the whole spectral range. The $\sim 2350 \mathrm{~cm}^{-1}$ band (*) in the L1 spectrum (a) is due to a slight variation of gaseous $\mathrm{CO}_{2}$ concentration in the purge inside the detector chamber.

The central $7.45 \mathrm{eV}$ energy $(167 \mathrm{~nm})$ for the CPL irradiations corresponds to a maximum in the absorption of RCPL (respectively LPCL) in the circular dichroism (CD) spectrum of several L (respectively D) amino acids, including alanine, dissolved in hexafluoroisopropanol (Fig. 2a, adapted from Snyder et al. 1973), due to the $\pi \pi^{*}$ transition of the carbonyl $\mathrm{C}=\mathrm{O}$ chromophore. The main goal of this study was to search for a possible enantiomeric excess, at room temperature, in the residues produced by UV CPL photo-processing of the ice. This energy is however different from the one used for the previous irradiation of pure leucine in solution with CPL leading to a small but significant enantioselective photo-destruction (Flores et al. 1977; Bonner \& Bean 2000). We searched for a similar or opposite effect during the photo-synthesis of chiral amino acids with CPL irradiation at $167 \mathrm{~nm}$.

The remaining gas mixtures irradiated in the $\mathrm{L} 1$ and L2 experiments were then used to perform similar deposition/irradiation experiments at the IAS (named I1 and I2, respectively) using the $\mathrm{H}_{2}$ discharge UV lamp during the same irradiation times as in the L1 and L2 experiments, respectively. Such irradiation is assumed to produce racemic residues since the UV light emitted by the $\mathrm{H}_{2}$ plasma is unpolarized (Muñoz Caro et al. 2002; Bernstein et al. 2002; Nuevo et al. 2005). Thus, we could compare the effects of both unpolarized and circularly polarized lights on the same starting ice mixtures in order to quantify the contribution of CPL to the stereochemistry of the amino acids produced in our residues.

The gas deposition and evolution of the ices during irradiation were controlled in-situ by a Fourier-transform infrared (FTIR) spectrometer (Bruker Vector 22) connected to the vacuum system, in the $4000-1000 \mathrm{~cm}^{-1}(2.5-10 \mu \mathrm{m})$ range $\left(2 \mathrm{~cm}^{-1}\right.$ resolution). After irradiation, the samples were slowly warmed up to room temperature to avoid any loss of the residue by explosive desorption of the ices due to exothermic radical recombination (d'Hendecourt et al. 1982). When the system reached thermal equilibrium at room temperature, the $\mathrm{MgF}_{2}$ windows covered with the residues were carefully removed, and IR spectra were recorded ex-situ using a Nicolet Magna-IR 560 ESP FTIR spectrometer coupled to a Nicolet Nicplan IR microscope $\left(2 \mathrm{~cm}^{-1}\right.$ resolution). Finally, the residues were kept under static
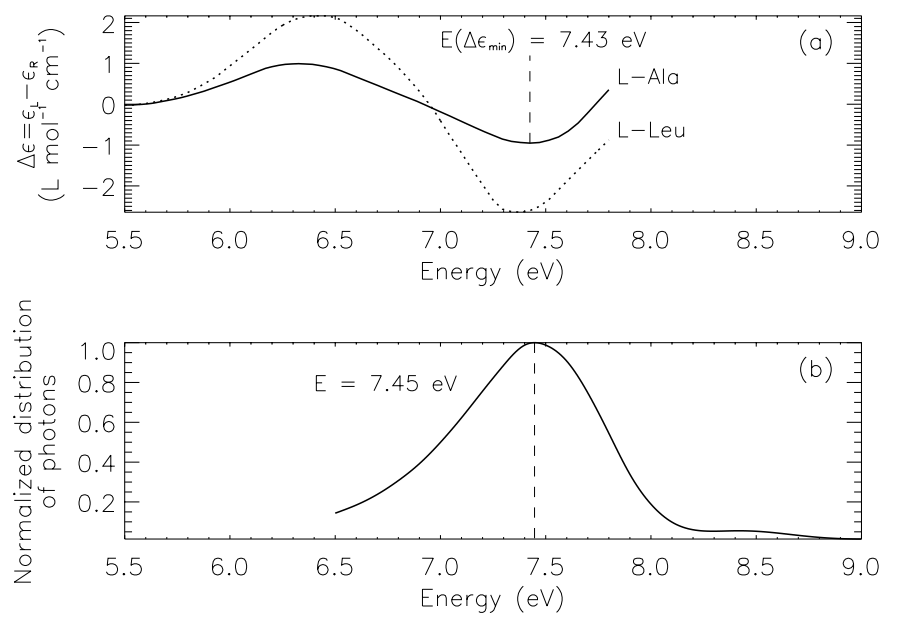

Fig. 2. Comparison between a) the CD spectra of L-alanine and L-leucine in solution (adapted from Snyder et al. 1973), and b) the SU5 UV CPL white beam at LURE (here for L1 experiment). In the CD spectra (a), $\epsilon_{\mathrm{L}}$ and $\epsilon_{\mathrm{R}}$ are the molar absorption coefficients for LCPL and RCPL, respectively. The energy of the synchrotron beamline $(7.45 \mathrm{eV})$ was chosen because of its overlap with the $\mathrm{C}=\mathrm{O}$ chromophore $\pi \pi^{*}$ transition of alanine and other amino acids in solution.

vacuum $\left(\sim 10^{-4}\right.$ mbar $)$ in order to avoid any kind of contamination or oxidation before being analysed by chemical techniques.

\subsection{Analyses of the organic residues at room temperature}

The general protocol for the chemical analyses of the amino acids with gas chromatography coupled to mass spectrometry (GC-MS) is described in Abe et al. (1996). This derivatization method allows free amino acids to become volatile and provides their separation in the chromatography column, without affecting their chiral ( $\mathrm{L}$ or D) configuration.

The residues were first extracted from their $\mathrm{MgF}_{2}$ window with $3 \times 30 \mu \mathrm{L}$ of $\mathrm{H}_{2} \mathrm{O}$ (grade: organic trace analysis, Fluka) using a sterilized Eppendorf pipette and placed in sterilized vials. In addition, a clean vial containing $3 \times 30 \mu \mathrm{L}$ of the same water underwent the same treatment as the residues (procedural blank). Then the water was evaporated by placing the vials in a desiccator (pressure $\sim 7$ torr). Once the water had totally evaporated, the samples were hydrolysed in $300 \mu \mathrm{L}$ of $6 \mathrm{M} \mathrm{HCl}$ (Fluka) and kept for $24 \mathrm{~h}$ in an oil bath maintained at $110{ }^{\circ} \mathrm{C}$. During this step, peptides and/or amino acid precursors are converted into free amino acids. Then $\mathrm{HCl}$ was evaporated in the desiccator.

The samples were then dissolved in $50 \mu \mathrm{L}$ of $0.1 \mathrm{M} \mathrm{HCl}$ and transferred into a reaction vial ( $1 \mathrm{~mL}$ volume). $25 \mu \mathrm{L}$ of an ethanol:pyridine $=3: 1$ mixture and $5 \mu \mathrm{L}$ of ethyl chloroformate EtOCOCl were added to the samples to derivatize the carboxylic acid and amino groups. Each vial was capped tightly and shaken vigorously for $10 \mathrm{~s}$. Immediately $N$-ethoxycarbonyl ethyl esters (ECEE) derivatives of amino acids were formed (Fig. 3). Then $15 \mu \mathrm{L}$ of chloroform were added and each vial was shaken for 2 min to extract the derivatives into the (lower) organic phase.

Finally, for each sample, $1 \mu \mathrm{L}$ of the organic phase was withdrawn by a microlitre syringe and injected directly into the gas chromatography Agilent 6890/5973 GC-MSD system equipped with a $25 \mathrm{~m}$ chiral stationary Chirasil-L-Val phase column (0.25 mm inner diameter, Varian-Chrompack). Splitless injections were performed, with an oven temperature programmed to $0 \mathrm{~min}$ at $50{ }^{\circ} \mathrm{C}$, and heated with $10{ }^{\circ} \mathrm{C} \mathrm{min}^{-1}$ to $90{ }^{\circ} \mathrm{C}, 2{ }^{\circ} \mathrm{C} \min ^{-1}$ to $110{ }^{\circ} \mathrm{C}$, and $10{ }^{\circ} \mathrm{C} \min ^{-1}$ to $180{ }^{\circ} \mathrm{C}$, 


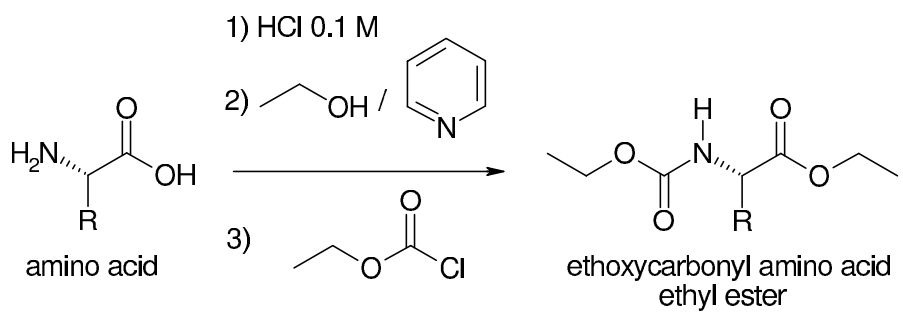

Fig. 3. Global equation for the derivatization of amino acids into ethoxycarbonyl ethyl ester (ECEE) derivatives for their separation with the GC-MS chiral column. Each compound keeps its initial chiral (L or D) configuration during the derivatization step.

where it was kept constant for $21 \mathrm{~min}$. Helium was used as a carrier gas with a constant flow of $1.5 \mathrm{~mL} \mathrm{~min}^{-1}$. In the scan mode of the mass spectrometer the fragmentation of each amino acid ECEE derivative was characterized by comparison with literature data (Huang et al. 1993; Muñoz Caro et al. 2002). The irradiated sample chromatograms were recorded in the more sensitive single-ion monitoring (SIM) mode of the mass spectrometer at the most intense ion fragments, e.g. $m / z=118$ a.m.u. and $m / z=177$ a.m.u. for ${ }^{13} \mathrm{C}$-Ala-ECEE and ${ }^{13} \mathrm{C}$-DAP-ECEE derivatives, respectively (see Sect. 3.2).

\section{Results}

\subsection{Infrared spectroscopy of the residues}

Figure 4 shows the IR spectra obtained for the residue produced during the LCPL irradiation run (L2) and the corresponding IAS residue (I2) irradiated with unpolarized light, recorded at room temperature with the IR microscope. Both spectra show striking similarities. They mainly consist of a broad band ranging from 3600 to $2500 \mathrm{~cm}^{-1}$ and of a clump of sharp features ranging from 1750 to $1150 \mathrm{~cm}^{-1}$, as already observed previously (Muñoz Caro \& Schutte 2003, and references therein). Only the relative intensities between the different features vary. This observation confirms that the processes with circularly polarized and unpolarized UV light sources were similar and significantly comparable. However, because of the $\mathrm{MgF}_{2}$ window IR cut-off, no information could be obtained for wavenumbers smaller than $\sim 1150 \mathrm{~cm}^{-1}$.

The identified IR bands in the spectra of both residues are reported in Table 1. Many chemical molecular groups could be identified: carboxylic acids and salts, alcohols, amines, alkane chains, esters and amides. We could also identify features ascribed to the ammonium ion $\mathrm{NH}_{4}^{+}$, already identified in 1950 by Wagner \& Horning, and different features ascribed to hexamethylenetetramine (HMT), a molecule first identified as a component of the residue produced from the photolysis of a $\mathrm{H}_{2} \mathrm{O}: \mathrm{CO}: \mathrm{NH}_{3}=5: 5: 1$ ice mixture (Briggs et al. 1992). HMT is also the most abundant component in residues when $\mathrm{CO}$ is substituted by methanol in the starting ice mixtures (Bernstein et al. 1995; Muñoz Caro \& Schutte 2003; Muñoz Caro et al. 2004), and only forms after warming the irradiated ices up to room temperature (Muñoz Caro \& Schutte 2003).

As expected, all the organic molecules detected were found to be ${ }^{13} \mathrm{C}$-labelled, since ${ }^{13} \mathrm{C}$-methanol was the unique source of carbon in our mixtures, and all infrared bands involving carbon atoms were shifted in the IR spectra. Therefore, we found no evidence for biological contamination in our experiments, which would have led to ${ }^{12} \mathrm{C}$-labelled products. Infrared data for ${ }^{13} \mathrm{C}$-labelled complex organic molecules are not easily available in the literature, in particular for molecules such as HMT.

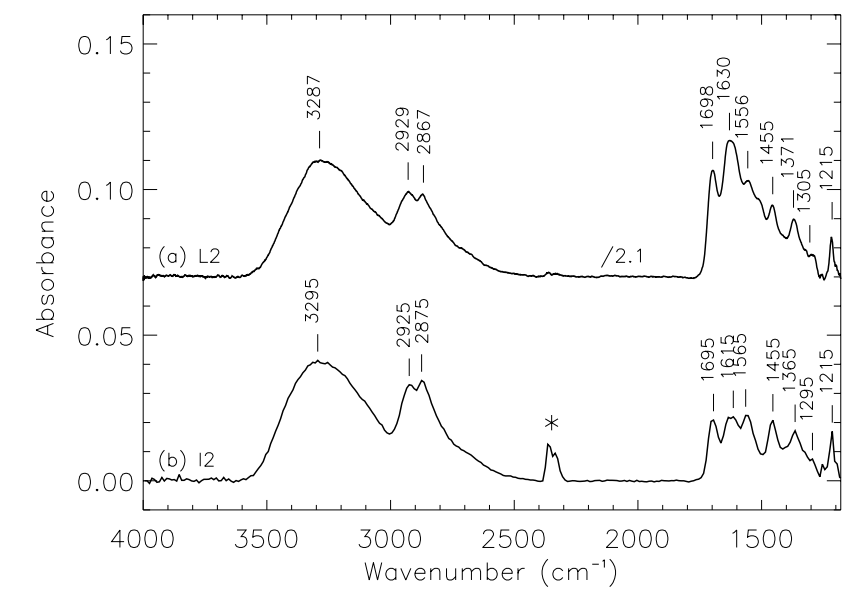

Fig. 4. Infrared spectra of L2 and I2 residues produced from the same $\mathrm{H}_{2} \mathrm{O}:{ }^{13} \mathrm{CH}_{3} \mathrm{OH}: \mathrm{NH}_{3}=1: 1: 1$ starting ice mixture, recorded with the IR microscope at room temperature: a) synchrotron irradiated L2 sample (LCPL, LURE), and b) $\mathrm{H}_{2}$ lamp irradiated I2 sample (unpolarized light, IAS). L2 residue spectrum (a) is divided by a factor 2.1 and offset for clarity. The IR band identifications are reported in Table 1 . As mentioned in Fig. 1, the $\sim 2350 \mathrm{~cm}^{-1}$ band $(*)$ in the IAS spectrum (b) is due to the purge.

So we estimated the shifted frequencies $v_{13}$ for these ${ }^{13} \mathrm{C}$ compounds from the known ${ }^{12} \mathrm{C}$-molecule frequencies $v_{12}$ using a simple harmonic oscillator model for molecular vibrations, and assuming that the vibration spring constants were close for ${ }^{12} \mathrm{C}$ and ${ }^{13} \mathrm{C}$ vibrations. The calculated shifted frequencies and the $v_{12} / v_{13}$ factors are reported in Cols. 4 and 5 of Table 1 , respectively. The comparison between the calculated frequencies and those reported from the IR spectra shows that this approximation is good and that the identifications are relevant for most of the molecular features, in particular for ${ }^{13} \mathrm{C}$-HMT. Moreover, the calculated shifts for the $\sim 1560$ and $\sim 1300 \mathrm{~cm}^{-1}$ bands, ascribed to carboxylic acid salts (Muñoz Caro \& Schutte 2003) are compatible with the shifts observed in the spectra of our ${ }^{13} \mathrm{C}$-labelled residues, confirming the identification of these bands.

Only HMT and $\mathrm{NH}_{4}^{+}$species could be identified in the residues from infrared data. The other IR features are due to molecular groups such as alcohols, amines or carboxylic acids. Thus, the IR spectra confirm the presence of a large variety of complex organic molecules, but do not allow us to deduce the presence of any specific amino acid because of their small relative abundances compared to other compounds. Therefore, exsitu chemical analyses are required.

\subsection{Gas chromatography coupled with mass spectrometry (GC-MS)}

The GC-MS analyses allowed us to clearly identify the amino acids that were present in our residues after extraction. The total ion-current (TIC) chromatograms obtained for L1 and I1 samples are given in Fig. 5, and show a large variety of amino acids in the residues. The peaks were mainly identified from I1 chromatogram (Fig. 5b), since the baseline and signal-to-noise ratio were better than for the L1 chromatogram. All peaks could not be identified. The mass fragments and retention time of each identified compound are reported in Table 2, where the amino acids given in boldface (alanine and DAP) are those for which the enantiomeric excesses were calculated and whose mass spectra are shown in Fig. 6 (I1 sample). 
Table 1. Feature identifications in L2 and I2 residues infrared spectra, produced from the same $\mathrm{H}_{2} \mathrm{O}:{ }^{13} \mathrm{CH}_{3} \mathrm{OH}: \mathrm{NH}_{3}=1: 1: 1$ ice mixture. The identified compounds are similar to those found in the residues produced and analysed by Muñoz Caro \& Schutte (2003). The expected positions in Col. 4 were estimated from calculations explained in the text, using the $v_{12} / v_{13}$ factors of Col. 5 .

\begin{tabular}{|c|c|c|c|c|}
\hline $\begin{array}{l}\text { Positions } \\
\left(\mathrm{cm}^{-1}\right)\end{array}$ & $\begin{array}{c}\text { Molecules or } \\
\text { molecular groups }\end{array}$ & $\overline{\text { Vibration modes }}$ & $\begin{array}{c}\text { Expected } \\
\text { positions }\left(\mathrm{cm}^{-1}\right)\end{array}$ & $\begin{array}{l}v_{12} / v_{13} \\
\text { factors }\end{array}$ \\
\hline $3500-2900$ & alcohols and amines & $\mathrm{O}-\mathrm{H} \& \mathrm{~N}-\mathrm{H}$ str. & $3380-3265$ & - \\
\hline (broad) & carboxylic acids & $\mathrm{O}-\mathrm{H}$ str. & $3100-2800$ & - \\
\hline $2929-2925$ & $\begin{array}{l}{ }^{13} \mathrm{C} \text {-alkane chains } \\
{ }^{13} \mathrm{C}-\mathrm{HMT}^{a}\end{array}$ & $\begin{array}{c}{ }^{13} \mathrm{CH}_{3} \text { and }{ }^{13} \mathrm{CH}_{2} \text { asym. str. } \\
\qquad v_{19}, v_{2}+v_{19}\end{array}$ & 2923-2921 & $1.001-1.002$ \\
\hline $2875-2867$ & $\begin{array}{c}{ }^{13} \mathrm{C} \text {-alkane chains } \\
{ }^{13} \mathrm{C}-\mathrm{HMT}^{a} \\
\mathrm{NH}_{4}^{+b}\end{array}$ & $\begin{array}{c}{ }^{13} \mathrm{CH}_{3} \text { and }{ }^{13} \mathrm{CH}_{2} \text { sym. str. } \\
v_{18} \text { sym. }{ }^{13} \mathrm{CH}_{2} \text { str. } \\
2 v_{4} \mathrm{NH} \text { str. }\end{array}$ & $2873-2871$ & $1.001-1.002$ \\
\hline $1698-1695$ & ${ }^{13} \mathrm{C}$-esters & ${ }^{13} \mathrm{C}=\mathrm{O}$ str. & 1703 & 1.023 \\
\hline $1630-1615$ & $\begin{array}{l}{ }^{13} \mathrm{C} \text {-ketones } \\
{ }^{13} \mathrm{C} \text {-amides }\end{array}$ & $\begin{array}{l}{ }^{13} \mathrm{C}=\mathrm{O} \text { str. } \\
{ }^{13} \mathrm{C}=\mathrm{O} \text { str. }\end{array}$ & $\begin{array}{l}1632 \\
1643\end{array}$ & $\begin{array}{l}1.023 \\
1.023\end{array}$ \\
\hline $\begin{array}{c}1565-1556 \\
1455\end{array}$ & $\begin{array}{c}{ }^{13} \mathrm{C} \text {-carboxylic acid salts }{ }^{c} \\
\mathrm{NH}_{4}^{+b}\end{array}$ & $\begin{array}{c}{ }^{13} \mathrm{COO}^{-} \text {asym. str. } \\
v_{4}\end{array}$ & 1561 & 1.016 \\
\hline $1371-1365$ & $\begin{array}{l}{ }^{13} \mathrm{C} \text {-alkenes } \\
{ }^{13} \mathrm{C}-\mathrm{HMT}^{a}\end{array}$ & $\begin{array}{l}{ }^{13} \mathrm{C}={ }^{13} \mathrm{C} \text { str. } \\
{ }^{13} \mathrm{CH} \text { scis. }\end{array}$ & $\begin{array}{l}1368 \\
1371\end{array}$ & $\begin{array}{l}1.041 \\
1.003\end{array}$ \\
\hline $\begin{array}{c}1305-1295 \\
1215\end{array}$ & $\begin{array}{c}{ }^{13} \mathrm{C} \text {-carboxylic acid salts }{ }^{c} \\
{ }^{13} \mathrm{C}-\mathrm{HMT}^{a}\end{array}$ & $\begin{array}{c}{ }^{13} \mathrm{COO}^{-} \text {sym. str. } \\
v_{21}{ }^{13} \mathrm{C} \equiv \mathrm{N}\end{array}$ & $\begin{array}{l}1299 \\
1210\end{array}$ & $\begin{array}{l}1.016 \\
1.021\end{array}$ \\
\hline
\end{tabular}

${ }^{a}$ Bernstein et al. (1995); ${ }^{b}$ Wagner \& Hornig (1950); ${ }^{c}$ Muñoz Caro \& Schutte (2003).

The abundances of the amino acids are found to decrease with increasing molecular complexity. Glycine (peak d) is the most abundant amino acid detected in the residues (Fig. 5), and then alanine $([\mathrm{Gly}] /[\mathrm{D}, \mathrm{L}-\mathrm{Ala}]) \simeq 3$ ), for which $\mathrm{D}$ and L enantiomers could be clearly separated by the column (peaks $\mathrm{b}$ and c, respectively). 2,3-Diaminopropanoic acid (DAP) is the most abundant non-proteinaceous chiral amino acid identified. Its enantiomers could also be well separated in the GC-MS SIM-mode. The highest peak of the chromatograms in Fig. 5 (peak i) is not an amino acid, but a derivatization byproduct.

The mass spectra of alanine and DAP enantiomers for the I1 sample (between 30 and 250 a.m.u.) are shown in Fig. 6. Each enantiomer of ECEE derivative was identified from its most intense peaks by comparison to literature data (Huang et al. 1993; Muñoz Caro et al. 2002). The mass spectra were obtained after removing the peaks due to known contaminants, such as phthalate $(m / z=149$ a.m.u.) from vinyl gloves.

\subsection{Determination of the enantiomeric excesses}

In order to determine the alanine and DAP e.e.s we needed to quantify the abundance of each enantiomer of Ala or DAP produced in each residue, at least their relative proportions, since $\mathrm{L}$ and $\mathrm{D}$ excesses, respectively $e e_{\mathrm{L}}$ and $e e_{\mathrm{D}}$, are defined by:

$e e_{\mathrm{L}}=\frac{L-D}{L+D} \quad$ and $\quad e e_{\mathrm{D}}=\frac{D-L}{L+D}=-e e_{\mathrm{L}}$,

where $L$ and $D$ are the quantities for $\mathrm{L}$ and $\mathrm{D}$ enantiomers, respectively. These quantities can be deduced from the GCMS chromatograms since the peak areas are in theory proportional to the amounts of material, with proportionality constants that are different for all compounds, even for the two enantiomers of the same molecule, due to their different interaction with the chiral column. Figure 7 shows the SIM chromatograms for 118 and 177 a.m.u., corresponding to ${ }^{13} \mathrm{C}$-Ala-ECEE and ${ }^{13} \mathrm{C}$-DAP-ECEE derivatives, respectively, recorded for L1 and I1 samples.

The most frequent problem for integrations is an overlap between $\mathrm{L}$ and $\mathrm{D}$ enantiomer peaks. So before integration, the peak
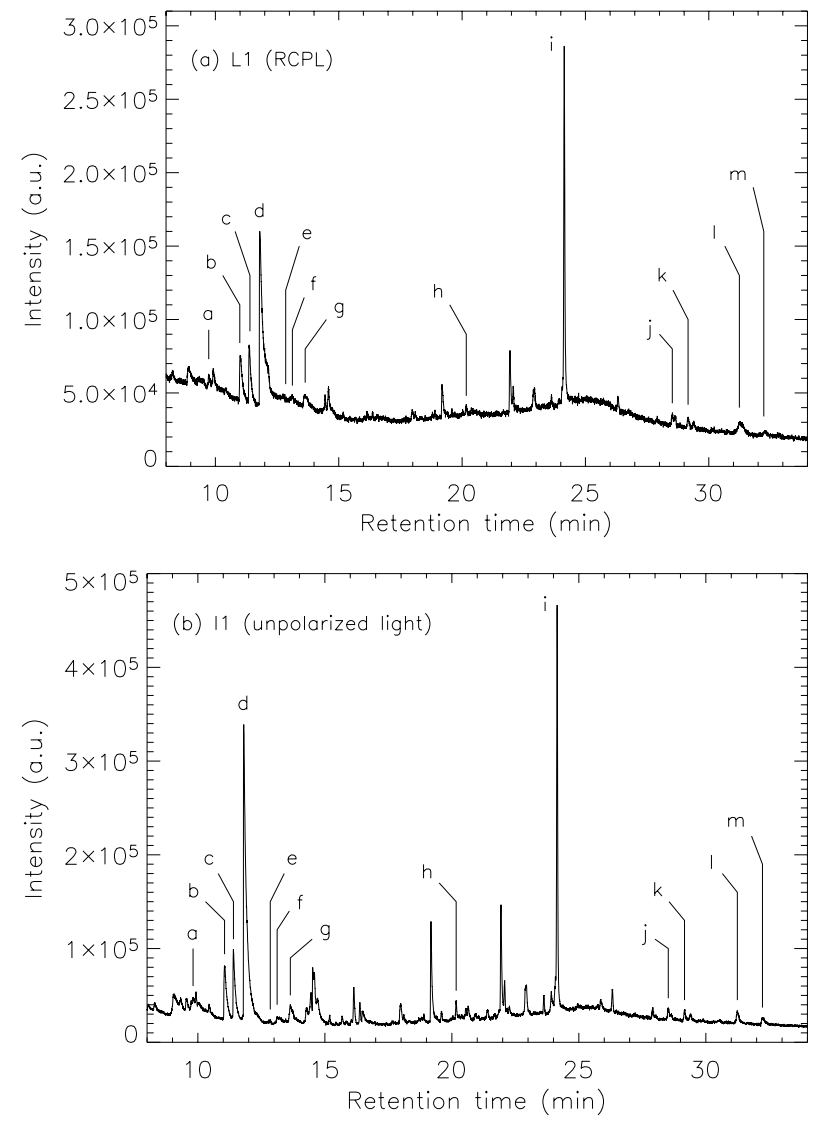

Fig. 5. Total ion-current (TIC) chromatograms of a) L1 (RCPL) and b) I1 (unpolarized light) samples, showing the large variety and complexity of amino acids or similar compounds produced. For I1 sample (b), the peak identification is reported in Table 2 and the mass spectra of the enantiomers of alanine (peaks b, c) and DAP (peaks j, k) are shown in Fig. 6.

curves were fitted using spline functions and interpolations. The fits obtained were very close to the chromatogram peaks and the tails hidden by overlapping were interpolated as closely as 
Table 2. Identification of the amino acids found in L1 and I1 residues from their fragment masses, corresponding to the TIC chromatogram shown in Fig. 5b (I1 sample, unpolarized light), listed by increasing retention time $R_{t}$ in the column. The amino acids given in boldface (alanine and DAP) are those from which the e.e.s were derived. The masses in boldface (Col. 3) correspond to the most intense peaks for each ECEE derivative ion, in atomic mass units (a.m.u.).

\begin{tabular}{clcc}
\hline \hline Peak & Amino acid & $\begin{array}{c}\text { MS-fragmentation for } \\
13 \text { C-samples (a.m.u.) }\end{array}$ & $R_{t}$ (min) \\
\hline a & Sarcosine (Sar) & $103, \mathbf{1 1 8}$ & 9.81 \\
b & D-Alanine (D-Ala) & $90,103, \mathbf{1 1 8}, 147$ & 11.05 \\
c & L-Alanine (L-Ala) & $90,103, \mathbf{1 1 8}, 147$ & 11.40 \\
d & Glycine (Gly) & $\mathbf{1 0 3}, 132,177$ & 11.81 \\
e & D-Aminobutyric acid & $103, \mathbf{1 3 3}$ & 12.85 \\
f & L-Aminobutyric acid & $103, \mathbf{1 3 3}$ & 13.12 \\
g & $\beta$-Alanine ( $\beta$-Ala) & $101,103, \mathbf{1 1 7}$ & 13.64 \\
h & D, L-Aspartic acid (D, L-Asp) & $\mathbf{1 9 1}$ & 20.17 \\
i & Derivatization by-product & $91,99, \mathbf{1 3 5}, 137$ & 24.14 \\
j & D-2,3-Diaminopropanoic acid (D-DAP) & $103,131,159, \mathbf{1 7 7}$ & 28.52 \\
k & L-2,3-Diaminopropanoic acid (L-DAP) & $103,131,159, \mathbf{1 7 7}$ & 29.16 \\
l & 3,3'-Diaminoisobutyric acid (DAIB) & $103,117, \mathbf{1 4 5}, 191$ & 31.24 \\
m & 1,2,3-Triaminopropane* & $\mathbf{1 0 3 , 1 1 7 , 1 3 2}$ & 32.23 \\
\hline
\end{tabular}

${ }^{*}$ Not an amino acid.
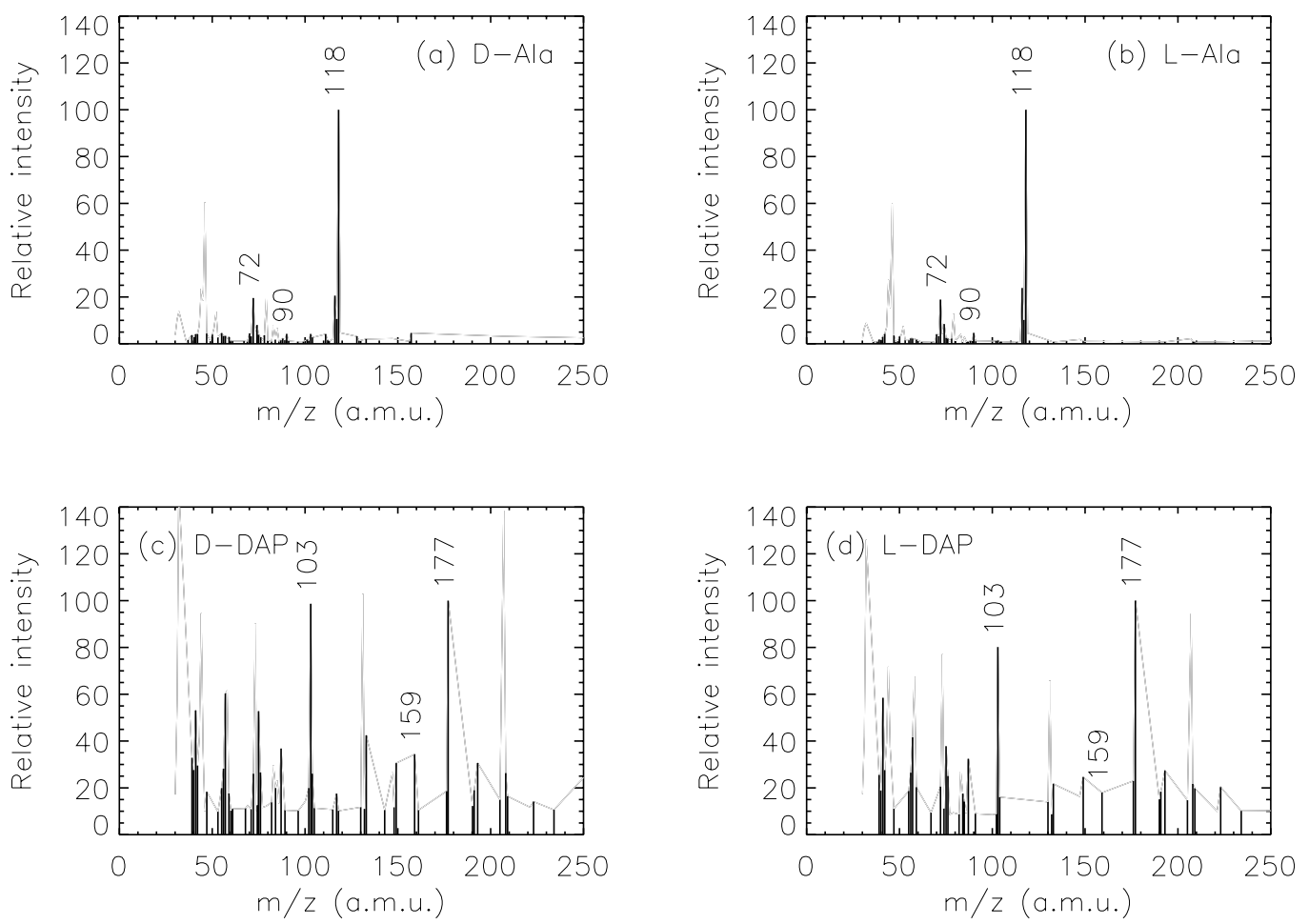

Fig. 6. Mass spectra (from 30 to 250 a.m.u.) of the identified enantiomers of alanine: a) D-Ala and b) L-Ala, and DAP: c) D-DAP and d) L-DAP, in I1 sample, corresponding to the peaks b, $c$ and j, k, respectively, in the TIC chromatogram of Fig. 5b. They were obtained after removing the peaks due to known contaminants, e.g. phthalate $(\mathrm{m} / \mathrm{z}=149$ a.m.u.). The most intense peaks for each molecule, corresponding to their most abundant ion fragments, were found to be similar to those found by Huang et al. (1993) and Muñoz Caro et al. (2002).

possible to the expected natural exponential decays. The enantiomeric excesses for alanine in L1/I1 and L2/I2 samples and for DAP in L1 and I1 samples, deduced from the calculated areas, are reported in Table 3 (Col. 5). The integration areas are given in Cols. 3 and 4.

The results in Table 3 show that the e.e.s calculated for I1 and I 2 samples, where the ices were irradiated with unpolarized light, are not zero. Theoretically, unpolarized light should lead to the production of a racemic mixture, i.e. $e e_{\mathrm{L}}=0$. This apparent excess is due to the fact that the interaction with the stationary phase of the chiral column is not exactly the same for the two enantiomers. Thus, a racemic sample injected into the column will display an apparent non-zero excess. Let us consider the chromatogram of a mixture supposed to be racemic, such as in the IAS samples. In theory, the $\mathrm{L}$ enantiomeric excess $e e_{\mathrm{L}}^{\text {th }}$ we should derive from the peak areas is zero:

$e e_{\mathrm{L}}^{\mathrm{th}}=\frac{L_{\mathrm{th}}-D_{\mathrm{th}}}{L_{\mathrm{th}}+D_{\mathrm{th}}}=0$

where $L_{\mathrm{th}}$ and $D_{\mathrm{th}}$ are the theoretical areas of the L and D enantiomers, respectively, if the interaction with the column were the same for both. However, as shown in Table 3, the two enantiomer peaks do not display the same area for I1 and I 2 samples, leading to an apparent non-zero measured excess. 
Table 3. Enantiomeric excesses for Ala in L1/L2 (UV CPL), and I1/I2 (UV unpolarized light) samples, and for DAP in L1 and I1 samples. These $\mathrm{L}$ excesses were deduced from integration of the peak areas (Cols. 3 and 4) of the SIM chromatograms (Fig. 7). The corrected e.e.s are given in Col. 7, after correcting the highest peak areas by the $\beta$ factors calculated from I1 and I2 samples and given in Col. 6.

\begin{tabular}{lcccccc}
\hline \hline $\begin{array}{l}\text { Polarization } \\
\text { (Amino acid) }\end{array}$ & Sample & $\begin{array}{c}\text { D peak area } \\
\pm 1 \sigma \text { error }\end{array}$ & $\begin{array}{c}\text { L peak area } \\
\pm 1 \sigma \text { error }\end{array}$ & $e e_{\mathrm{L}}$ & $\beta$ factors & Corrected $e e_{\mathrm{L}}$ \\
\hline RCPL (Ala) & L1 & $3411559 \pm 6269$ & $3547689 \pm 7371$ & $+1.96 \%$ & & $+1.10 \%$ \\
unpolarized (Ala) & I1 & $8321042 \pm 14910$ & $8464743 \pm 17586$ & $+0.86 \%$ & 1.017 & $+0.40 \%$ \\
\hline LCPL (Ala) & L2 & $3640.5 \pm 8.1$ & $3747.2 \pm 8.4$ & $+1.44 \%$ & & $-0.85 \%$ \\
unpolarized (Ala) & I2 & $3111.1 \pm 7.2$ & $3176.7 \pm 7.6$ & $+1.04 \%$ & 1.021 & \\
\hline RCPL (DAP) & L1 & $299311 \pm 1199$ & $306139 \pm 1091$ & $+1.13 \%$ & & 1.040 \\
unpolarized (DAP) & I1 & $571303 \pm 2099$ & $594372 \pm 1965$ & $+1.98 \%$ & & \\
\hline
\end{tabular}
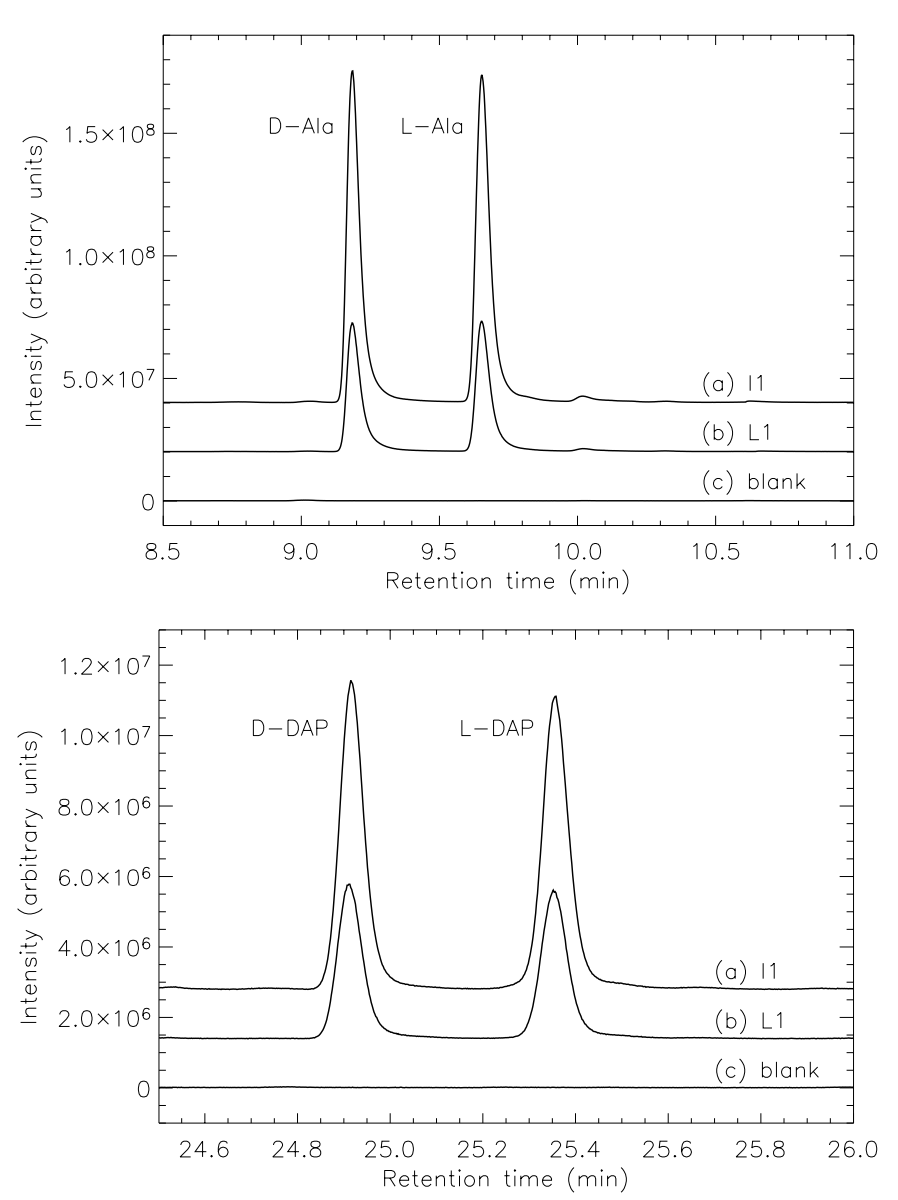

Fig. 7. SIM-mode GC-MS chromatograms of a) I1 and b) L1 samples for 118 a.m.u. ( ${ }^{13} \mathrm{C}$-Ala-ECEE derivative, top) and 177 a.m.u. $\left({ }^{13} \mathrm{C}\right.$ DAP-ECEE derivative, bottom) with a chiral Chirasil-L-Val column. Alanine was found to be $\sim 2.4$ times and DAP $\sim 1.9$ times more abundant in I1 than in L1. This is probably due to the fact that during CPL irradiation less than $25 \%$ of the window surface was irradiated (see Sect. 2.1). The (c) chromatograms correspond to a blank sample prepared by irradiating a $\mathrm{MgF}_{2}$ window at IAS with unpolarized light, without any deposition, and following the same extraction protocol as the residues. They clearly demonstrate that no contamination affects the chromatograms.

Let $\beta$ be a dimensionless factor multiplying the peak having the greater area, here the L peak, and corresponding to the enhancement of the column response for this enantiomer $(\beta \geq 1)$. The measured non-zero excess for the I1 and I 2 samples would thus be defined by:

$e e_{\mathrm{L}}^{\mathrm{IAS}}=\frac{L_{\mathrm{IAS}}-D_{\mathrm{IAS}}}{L_{\mathrm{IAS}}+D_{\mathrm{IAS}}}=\frac{\beta L_{\mathrm{th}}-D_{\mathrm{th}}}{\beta L_{\mathrm{th}}+D_{\mathrm{th}}} \neq 0$, where $L_{\text {IAS }}$ and $D_{\text {IAS }}$ are the measured areas in I1 and I 2 samples, for $L$ and $D$ peaks, respectively. In this case, the area measured for the D peak would be equal to the theoretical area: $D_{\mathrm{IAS}}=D_{\mathrm{th}}$. From Eq. (2), we set $L_{\text {th }}=D_{\text {th }}$ and finally obtain:

$e e_{\mathrm{L}}^{\mathrm{IAS}}=\frac{\beta-1}{\beta+1} \Rightarrow \beta=\frac{L_{\mathrm{IAS}}}{D_{\mathrm{IAS}}}$.

Therefore, to correct the effect of the column for the LURE samples and quantify the effect of CPL with respect to unpolarized light, we have to correct the peak areas $L_{\mathrm{LURE}}$ measured for L enantiomers in the L1 and L2 samples with the $\beta$ factors:

$e e_{\mathrm{L}}^{\mathrm{LURE}}=\frac{L_{\mathrm{LURE}} / \beta-D_{\mathrm{LURE}}}{L_{\mathrm{LURE}} / \beta+D_{\mathrm{LURE}}}$,

where $L_{\text {LURE }}$ and $D_{\text {LURE }}$ are the L and D peak areas measured in the LURE samples, respectively. This method is valid since for each couple of experiment, L1/I1 and L2/I2, the samples produced from CPL and unpolarized light irradiations were extracted from their $\mathrm{MgF}_{2}$ windows, hydrolysed and derivatized at the same time, to be injected one after the other into the same column, so the conditions of the column, and thus the $\beta$ factors, were similar for both injections. The results taking those corrections into account are reported in Cols. 6 ( $\beta$ factors derived from I1 and 12 samples) and 7 (corrected $e e_{\mathrm{L}}$ for L1 and L2 samples) of Table 3, where we can see that the corrected e.e.s actually correspond to the subtraction $e e_{\mathrm{L}}^{\mathrm{LURE}}-e e_{\mathrm{L}}^{\mathrm{IAS}}$.

The second and certainly most important result of Table 3 is that the reported excesses are small, with an upper limit of $\sim 1 \%$ for Ala and DAP in absolute values (corrected e.e.s for L1 sample). Therefore, the main result of these experiments is that the magnitude of the enantiomeric excesses induced by CPL irradiation of ices at $167 \mathrm{~nm}$ on the photoproducts is small. Finally, the signs of the calculated e.e.s are not those expected in each experiment: alanine displays L excesses with both polarizations, whereas DAP was found to display a D excess with RCPL, opposed to the $\mathrm{L}$ excess found for Ala. The significance of these e.e.s will be discussed in Sect. 4 after the determination of the associated uncertainties.

\subsection{Estimate of the error bars and final excesses}

A careful estimate of the error bars is essential since it has repercussions for the signal-to-noise ratios of the chromatograms, which play an important role in the data analysis. We could identify three main sources leading to uncertainties in the determination of enantiomeric excesses. There may be other sources of error, but they are not easy to identify and estimate.

1. Intrinsic effect of the chiral column. When several injections of the same sample are performed, each chromatogram 
Table 4. Determination of the integration $\left(\sigma_{\beta_{\text {int }}}\right)$ and global $\beta$ factor uncertainties $\left(\sigma_{\beta}\right)$, calculated for each experiment with $\sigma_{\beta_{\text {col }}}<0.002$ (see Sect. 3.4). The corrected $e e_{\mathrm{L}}$, with their total $3 \sigma$ uncertainties (see Eq. (8)), are given in Col. 6.

\begin{tabular}{lccccc}
\hline \hline $\begin{array}{l}\text { Polarization } \\
\text { (Amino acid) }\end{array}$ & Sample & $e e_{\mathrm{L}}$ & $\sigma_{\beta_{\text {int }}}$ & $\sigma_{\beta}$ & $\begin{array}{c}\text { Corrected } e e_{\mathrm{L}} \\
\pm 3 \sigma \text { error }\end{array}$ \\
\hline RCPL (Ala) & $\mathrm{L} 1$ & $+1.96 \%$ & 0.0028 & 0.0033 & $+1.10 \pm 0.64 \%$ \\
unpolarized (Ala) & $\mathrm{I} 1$ & $+0.86 \%$ & & & \\
\hline LCPL (Ala) & $\mathrm{L} 2$ & $+1.44 \%$ & 0.0034 & 0.0038 & $+0.40 \pm 0.74 \%$ \\
unpolarized (Ala) & $\mathrm{I} 2$ & $+1.04 \%$ & & & \\
\hline RCPL (DAP) & $\mathrm{L} 1$ & $+1.13 \%$ & 0.0051 & 0.0054 & $-0.85 \pm 1.12 \%$ \\
unpolarized (DAP) & $\mathrm{I} 1$ & $+1.98 \%$ & & & \\
\hline
\end{tabular}

shows small differences compared to the others, of the order of $0.1 \%$. This can be because the concentration of the sample is not exactly the same for each injection, or can be due to column bleeding, i.e. contamination of the samples by molecules from the inside of the column itself, or to a difference of interaction of the sample with the column between two injections. These three effects will be considered as a global intrinsic effect of the column.

2. Coelution of contaminants. Compounds belonging to our samples may elute from the column with the same retention times as Ala or DAP. In that case, the peaks of the contaminant molecules in the chromatograms overlap with those of Ala and DAP and contribute to the peak areas for integration. Since these possible contaminants come from the samples themselves, they cannot be isotopically separated because they are also labelled with ${ }^{13} \mathrm{C}$. However, GC-MS technique allows compounds to be identified by both their retention time and their mass spectrum (see Sect. 3.2), avoiding undetected contaminants. This source of error is thus negligible as compared to the others, provided that (and only if) the technique used combines both GC and MS. This becomes an important source of error for classical GC.

3. Integration of the peaks. To calculate e.e.s, it is important to properly integrate the peaks, taking difficulties such as overlapping between peaks into account, which could be partly solved by fitting the peaks with spline functions after correction of the chromatogram baselines. The uncertainties on the calculated areas mainly come from the time resolution of the column $\delta t$, and deviations of the peak fits to the experimental curves, especially in overlapping intervals, and where the spline functions interpolate the peak tails.

As already mentioned, the coelution of contaminants seems to be an unlikely source of uncertainty in our calculations of the excesses. For the two other sources, we could make a reasonable estimate of their order of magnitude by studying the behaviour of the column for several injections of a similar sample analysed with the same column as the one used for L1, I1, L2 and I2 samples. So we could estimate how $\beta$ factors vary for a set of consecutive injections of the same sample (separated in time by about one hour), and derive a mean $\beta$ factor and a standard deviation $\sigma_{\beta_{\mathrm{col}}}$, corresponding to the uncertainty due to the intrinsic effect of the column.

The organic residue used for this study was obtained after irradiation of a $\mathrm{H}_{2} \mathrm{O}: \mathrm{CH}_{3} \mathrm{OH}: \mathrm{NH}_{3}=1: 1: 1$ ice mixture (no ${ }^{13} \mathrm{C}$ labelled methanol, $\mathrm{CH}_{3} \mathrm{OH}$ from Aldrich, $99.9 \%$ purity) with the $\mathrm{H}_{2}$ lamp at the IAS (unpolarized light), and was similar to L1, $\mathrm{I} 1, \mathrm{~L} 2$ and $\mathrm{I} 2$ residues. The only difference is that enough residue was produced to perform 6 independent injections in the GC-MS system after hydrolysis and derivatization.

The $\beta$ factors were calculated for the 6 injections after integration of the peaks, from which we derived a mean value of $\left\langle\beta_{\mathrm{col}, 6}\right\rangle=1.031$, with a standard deviation $\sigma_{\beta_{\mathrm{col}, 6}}<0.002$. However, since the two samples irradiated with CPL yielded small amounts of residue, only one injection could be performed for each mass (118 and 177 a.m.u. for Ala and DAP, respectively). Thus, a more significant and reliable value of $\sigma_{\beta_{\text {col }}}$ is given by the way the column behaves between two consecutive injections. To estimate it, we considered the 5 absolute differences $\delta_{i j}$ between the $\beta$ factors calculated for two consecutive $i$ and $j$ injections. Assuming that these values correspond to standard deviations, the $\sigma_{\beta_{\text {col }}}$ error we make on the calculation of $\beta$ between two injections is the mean $\delta_{i j}$ value. After calculation, we found that $\sigma_{\beta_{\text {col }}}<0.002$, which is similar to the 6-injection standard deviation $\sigma_{\beta_{\mathrm{col}, 6}}$ calculated before. This value is a quantification of the intrinsic effect of the column and will be used afterwards for the calculations of uncertainties.

The error on $\beta$ also comes from the integration of the $\mathrm{L}$ and D peaks of the IAS samples. This error $\sigma_{\beta_{\text {int }}}$ can be estimated using the general equation of error propagation approximation. Since $\beta$ is a function of the two variables $L_{\text {IAS }}$ and $D_{\text {IAS }}$ (Eq. (4)), we obtain:

$\sigma_{\beta_{\mathrm{int}}}^{2}=\frac{1}{D_{\mathrm{IAS}}^{2}}\left[\sigma_{L_{\mathrm{IAS}}}^{2}+\sigma_{D_{\mathrm{IAS}}}^{2}\left(\frac{L_{\mathrm{IAS}}}{D_{\mathrm{IAS}}}\right)^{2}\right]$.

The same way, the uncertainty $\sigma_{A}$ we make when integrating the areas $A$ for $\mathrm{L}$ and $\mathrm{D}$ enantiomers in each IAS chromatogram, assuming that the peak areas $A$ are proportional to their base width $\Delta t$ and their height $h$, is given by:

$\left(\frac{\sigma_{A}}{A}\right)^{2}=\left(\frac{\sigma_{h}}{h}\right)^{2}+\left(\frac{\sigma_{t}}{\Delta t}\right)^{2}$

where $\sigma_{h}$ is the uncertainty on the peak height $h$, equal to the mean noise around the considered peak, and $\sigma_{t}=\delta t=$ $2 \times 10^{-3} \mathrm{~min}$ is the time resolution of the column. The peak bases $\Delta t$ were taken within the spline-fitted intervals. The calculated areas and their associated uncertainties are reported in Cols. 3 and 4 of Table 3. The total $\beta$ factor uncertainties $\sigma_{\beta}$ are obtained quadratically: $\sigma_{\beta}^{2}=\sigma_{\beta_{\text {col }}}^{2}+\sigma_{\beta_{\text {int }}}^{2}$. The detailed and global uncertainties for all $\beta$ factors are given in Cols. 4 and 5 of Table 4.

Finally, using these global $\beta$ factors uncertainties, Eq. (5) and the general equation of error propagation approximation, the error $\sigma_{e e_{\mathrm{L}}^{\mathrm{LURE}}}$ made on the L excess for L1 and L2 samples is given by:

$$
\begin{array}{r}
\sigma_{e e_{\mathrm{L}}^{\mathrm{LURE}}}^{2}=\frac{4 \beta^{2}}{\left(L_{\mathrm{LURE}}+\beta D_{\mathrm{LURE}}\right)^{4}}\left[\sigma_{L_{\mathrm{LURE}}}^{2} D_{\mathrm{LURE}}^{2}\right. \\
\left.+\sigma_{D_{\mathrm{LURE}}}^{2} L_{\mathrm{LURE}}^{2}+\sigma_{\beta}^{2}\left(\frac{L_{\mathrm{LURE}} D_{\mathrm{LURE}}}{\beta}\right)^{2}\right],
\end{array}
$$

where $L_{\mathrm{LURE}}$ is the measured $\mathrm{L}$ peak area before correction. The final $e e_{\mathrm{L}}$ for the CPL experiments for Ala and DAP and their associated uncertainties are given in Col. 6 of Table 4 . These $3 \sigma$ 
uncertainties correspond to a probability of more than $99.7 \%$ for the values to be inside the interval of likelihood, assuming a Gaussian distribution of the values.

\section{Discussion}

\subsection{Enantioselective processes}

The corrected enantiomeric excesses of alanine and DAP in the L1 sample correspond to $5.1 \sigma$ and $2.3 \sigma$ measurements, respectively, and may thus be considered as significant to a first approximation. However, although these excesses were found to be of the same order of magnitude for both amino acids, they display opposite signs. Considering only the absolute values, the uncertainties determined for the excesses in the L1 residue (RCPL) for alanine and DAP are smaller than those for alanine in the L2 residue (opposite polarization, LCPL). That does not mean that RCPL is more efficient than LCPL, but it reveals that the L2 experiment did not work as well as L1. This can be due to several factors:

1. Racemization processes, inside the ice matrices and/or after extraction during the chemical analysis, in particular during the acid hydrolysis, which could have been different for each experiment (see text hereafter for more details).

2. The number of photons per molecule ratios, which is a factor 2 smaller in L2 than in L1 (Sect. 2.1), and that could lead to the production of less material and/or a less complex organic residue in $\mathrm{L} 2$.

3. A (partial) loss of the final product during the warm-up to room temperature (by explosive sublimation) and/or during the chemical analysis.

Since the L2 experiment gave a smaller amount of organic residue, it is not unexpected to find a bigger relative error for the $e e_{\mathrm{L}}$ derived for alanine. In this irradiation, one (or several) factor(s) among those mentioned above is (are) certainly responsible for the destruction or partial racemization of a fraction of the amino acids or their precursors, leading to GC-MS measurements with higher noise.

If we consider the results for the $\mathrm{L} 1$ sample, i.e. an L excess of $1.10 \%$ for Ala and a D excess of $0.85 \%$ for DAP, no conclusion can be drawn on the photo-processes occurring when ices are irradiated by UV CPL at $167 \mathrm{~nm}$. From Fig. 2, we can predict that, at this wavelength, L (respectively D) enantiomers of amino acids, or their chiral precursors, should mainly absorb RCPL (respectively LCPL). Thus, in the case of an enantioselective photo-destruction of the formed molecules, irradiation with RCPL (respectively LCPL) should lead to an enhancement of D (respectively L) enantiomers. This may be valid assuming that the interactions between the molecules inside the ice matrices - i.e. a medium relevant to astrophysical ices - and CPL are not significantly different from those affecting pure amino acids. In particular, the chiral molecules which are present in the ices should absorb UV light in the same range as pure amino acids.

However, the results reported in Tables 3 and 4 show excesses which are in disagreement with a selective photodestruction process, as observed in previous experiments (Flores et al. 1977; Bonner \& Bean 2000) where final excesses, after CPL irradiation at $212.8 \mathrm{~nm}$, were found to be in favour of the enantiomers absorbing the least. In the L1 sample, RCPL seems to induce an opposite effect on the two compounds, since Ala displays an L excess and DAP a D excess. Three main reasons could explain this result:

1. alanine and DAP underwent different (photo-)processes leading to totally opposite e.e.s;

2. although these amino acids were clearly identified by GC-MS, too little material was produced to quantify such a small effect;

3. both amino acids underwent racemization processes during the acid hydrolysis of the residues, but in different proportions (see text hereafter).

The CD spectra of most amino acids are expected to be similar in the energy range considered here, since they all contain the same $\mathrm{C}=\mathrm{O}$ chromophore responsible for the 168-nm absorption band (see Fig. 2a and Snyder et al. 1973), so that the hypothesis of a production of non-racemic amino acids with opposite e.e.s is unlikely. Therefore, the excesses found for alanine and DAP in L1 (RCPL) reveal that we are limited by the detection sensitivity of the GC-MS system, and that under our experimental conditions, the effect of UV CPL at $167 \mathrm{~nm}$ on the production of non-racemic organic molecules is weak and $\lesssim 1 \%$.

This detection limit implies that e.e.s smaller than $1 \%$ may not be reliable when only chromatography techniques are used for the detection of small quantities of material. This could explain the discrepancies observed in the excesses found for both amino acids and both polarizations. Moreover, the high relative uncertainty derived for Ala excess in L2 shows that such CPL irradiations must be repeated in order to increase the signal-tonoise ratio of the chromatograms, by producing more material and/or by improving the sensitivity of the instruments. The total UV fluence for one given experiment could also be increased. A larger photon flux will be available on the new synchrotron radiation beamline DESIRS at SOLEIL, now under construction in France. In future experiments the amount of material will thus be the main limiting parameter in the e.e. determination.

Finally, racemization processes can also play an important role in the final results. It is well known that all amino acids racemize, i.e. they lose their chiral purity with time. This process, which is also temperature- and $\mathrm{pH}$-dependent, is particularly efficient during the acid hydrolysis step, due to proton exchanges (Manning 1970; Bada 1972). It can be neglected for amino acids such as alanine, valine or leucine, but DAP was shown to racemize significantly during acid hydrolysis (Jacobson et al. 1974). In order to check these results, we hydrolysed pure alanine and DAP standards under the same conditions as L1, I1, L2 and I2 samples. The results show that, under these conditions, no racemization occurred with a hydrolysed standard sample of D-alanine, nor with a separately hydrolysed sample of L-alanine. However, a standard sample of L-DAP was found to racemize in a way that $31 \%$ of the hydrolysed L-DAP was converted into D-DAP. This may explain the contradictory results observed for the two amino acids studied.

\subsection{Comparison with other amino acid data}

Recently, experiments on the enantioselective photo-destruction of leucine in the solid phase with CPL were performed by Meierhenrich et al. (2005). They measured a circular dichroism spectrum for solid leucine films at room temperature, showing the same type of electronic spectrum as for leucine in solution but where the $\pi \pi^{*}$ transition is red-shifted in wavelength by $23 \mathrm{~nm}(0.89 \mathrm{eV})$. After irradiation of these films, they measured a maximal D excess of $2.60 \%$ with RCPL at $182 \mathrm{~nm}(6.81 \mathrm{eV})$. These new data, obtained in a phase state closer to our experimental conditions, will be used in future experiments. 


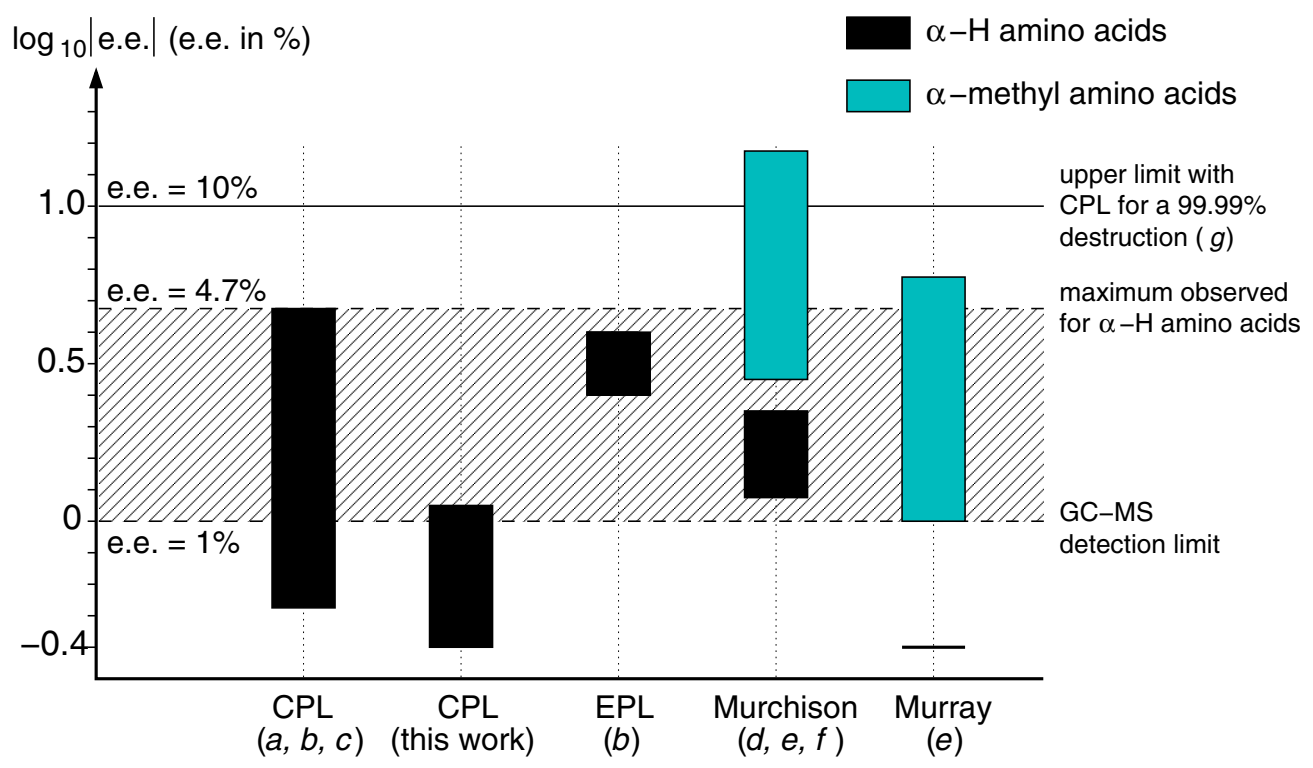

Fig. 8. Diagram summarizing all enantiomeric excesses for amino acids mentioned in this paper, for CPL and EPL experiments (including this work), and meteoritic analyses. The hatched area corresponds to the region where these different determinations seem to converge for $\alpha$-H amino acids, such as proteinaceous amino acids, taking the GC-MS detection limit discussed in this paper into account. References: $(a)$ Flores et al. (1977); (b) Bonner \& Bean (2000); (c) Meierhenrich et al. (2005); (d) Cronin \& Pizzarello (1997); (e) Cronin \& Pizzarello (1999); $(f)$ Pizzarello et al. (2003); $(g)$ Balavoine et al. (1974).

All these results, in particular those obtained in our RCPL experiment and in other experiments using polarized light (Flores et al. 1977; Bonner \& Bean 2000; Meierhenrich et al. 2005) must be compared with extraterrestrial material data, i.e. analyses of meteorites such as Murchison and Murray, where many amino acids were detected and found to display $\mathrm{L}$ enantiomeric excesses after chemical analyses (Cronin \& Pizzarello 1997, 1999). Figure 8 summarizes all the e.e.s obtained in experiments and measured in the Murchison and Murray meteorites.

In meteorites the highest e.e.s were found for nonproteinaceous amino acids, e.g. $8.4 \%$ for isovaline and $9.1 \%$ for $2 S, 3 R / 2 R, 3 S$-2-amino-2,3-dimethylpentanoic acid (DMPA) (Cronin \& Pizzarello 1999). Recent measurements on several Murchison meteorite samples also show that isovaline displays excesses ranging from 0 to $15.2 \%$ (Pizzarello et al. 2003). Some proteinaceous amino acids were also found to be non-racemic, but with smaller e.e.s: an excess of $2.2 \%$ was measured for valine, and $1.2 \%$ for alanine (Cronin \& Pizzarello 1999), which is actually a value close to the one found in our experiments. This last result has important consequences for the significance of such a small excess since it is very close to the detection limit we estimated (of the order of $1 \%$, see Sect. 4.1). Moreover, problems of coelution (see Sect. 3.4), not always clearly mentioned in the meteoritic amino acid analysis literature, could be responsible for errors in some measurements of enantiomeric excesses. Therefore, we would like to draw attention to the fact that small excesses should always be measured using at least two complementary and independent techniques of analysis (e.g. chromatography and mass spectrometry), and after a thorough study of the systematic effects, which dominate the signal-to-noise ratio in the chromatograms, and can lead to wrong apparent excesses.

In general, it seems that meteoritic $\alpha$-methyl ( $\alpha$-Me) amino acids, i.e. with a methyl group bound to the carbon just next to the carbonyl, display higher e.e.s than their $\alpha$-hydrogenated ( $\alpha$ $\mathrm{H})$ analogs. Actually, $\alpha-\mathrm{H}$ amino acids are known to racemize more efficiently than $\alpha$-Me amino acids (Pollock et al. 1975), because a $\mathrm{H}$ atom bound to a carbon close to a carbonyl is easy to remove. When an $\alpha$-H amino acid loses its $\alpha$ - $\mathrm{H}$ atom, the asymmetric carbon loses its chirality. The resulting plane geometry around this atom allows $\mathrm{H}$ atoms to interact with the molecule from either side with roughly the same probability, leading to a racemization of the final compound. This does not occur so easily with $\alpha$-Me amino acids, because methyl groups cannot be removed as easily as $\mathrm{H}$ atoms.

This could explain at least in part why, after the chemical treatment of meteoritic samples, proteinaceous amino acids, which are all $\alpha-\mathrm{H}$ compounds, were found to display excesses with factors up to 5 smaller than $\alpha$-Me amino acids (Cronin \& Pizzarello 1999), and why in our samples Ala and DAP, both $\alpha$ hydrogenated, were found to display very low excesses, close to our detection limit. The study of $\alpha$-Me amino acids produced by UV CPL irradiation of ices would thus be really interesting in the future, to check if such compounds display high e.e.s. Moreover, $\alpha$-Me amino acids such as isovaline were recently shown to be better asymmetric catalysts than $\alpha-\mathrm{H}$ amino acids, i.e. they can efficiently orientate chemical enantioselective reactions to the production of one type of enantiomer (Pizzarello \& Weber 2004), and thus they appear to be key compounds to study in order to elucidate their role in the origin of homochirality. However, $\alpha$-Me amino acids are difficult to study since they are produced in much lower abundances than $\alpha$-H compounds in the residues. Isovaline was identified in only one organic residue formed by unpolarized UV irradiation of a $\mathrm{CH}_{3} \mathrm{OH}: \mathrm{NH}_{3}=1: 1$ ice mixture (Nuevo et al. 2005). But in our CPL experiments, no $\alpha$-Me amino acid was detected, confirming their low production yield.

Finally, the high excesses measured for isovaline in some Murchison meteorite samples (Pizzarello et al. 2003) are also in disagreement with our results and previous experiments showing that the contribution of CPL cannot exceed the upper limit of $\sim 10 \%$ for a $99.99 \%$ decomposition of amino acids (Balavoine et al. 1974), assuming a realistic anisotropy factor $g$ 
of $\sim 0.02$ (Nishino et al. 2002). Therefore, homochirality cannot be a direct consequence of UV CPL irradiation of organic molecules in space, and amplification processes of small e.e.s (Brack \& Spach 1981; Shibata et al. 1998) in astrophysical environments or on the early Earth are needed to account for the observed homochirality. The best candidates for this amplification process are $\alpha$-methyl amino acids (Hough et al. 2001), since they are less sensitive to racemization processes (Pizzarello \& Cronin 2000).

\section{Conclusions}

These are the first reported results on enantiomeric excess quantifications for amino acids produced by irradiation of interstellar ice analogs with circularly polarized UV light. After a detailed data analysis and a careful estimate of the experimental uncertainties, we find that the effect of CPL at $7.45 \mathrm{eV}(167 \mathrm{~nm})$ on such ice mixtures is weak, i.e. the enantiomeric excesses measured for alanine and DAP are very small, close to $1 \%$ in absolute values, and opposite for alanine (L excess) and DAP (D excess). The detection limit for the measurement of such a small effect with GC-MS was also estimated and found to be $\sim 1 \%$. Therefore, these results cannot allow us to determine the process(es) leading to the formation of chiral molecules displaying such excesses at this wavelength.

To quantify more accurately the effect of CPL on such molecules, new irradiation experiments using synchrotron UV light will be performed, where much larger quantities of amino acids must be produced in order to detect, identify and quantify their enantiomers with GC-MS, and to lower the error bars on the derived excesses. Different wavelengths should also be chosen, taking the recent results obtained for solid leucine into account. This will be possible with the new VUV beamline DESIRS on the future synchrotron center SOLEIL in 2007, where future experiments may confirm - or not - the enantioselective photodestruction mechanism observed for pure amino acids.

Finally, our results were compared with the e.e.s measured for some meteoritic amino acids, which should however be considered with care as discussed in this work. The presence of these non-racemic amino acids in meteorites and their isotopic ratios tend to favour an extraterrestrial origin for non-racemic organics. Moreover, the observation of infrared CPL and its probable UV counterpart in the OMC-1 molecular cloud in Orion support an asymmetric photochemical origin of homochirality. However, the polarization rates for OMC-1 are much smaller than those mentioned in our CPL experiments. This could imply that the irradiation of icy mantles on the surface of cold grains by UV CPL in the ISM or the pre-solar cloud is not an efficient mechanism for the production of high enantiomeric excesses. An amplification process, starting for instance from $\alpha$-methyl amino acids, is therefore necessary to account for the observed homochirality.

Acknowledgements. We would like to thank J. M. Greenberg, who first had the idea for these CPL experiments. We are greatful to the PCMI and CNES for financial support. G. M. M. C. is grateful for a Marie Curie individual fellowship, and U. J. M. is grateful for a researcher's position, GC-MS equipment, and a research group funded by the Deutsche Forschungsgemeinschaft (DFG), Bonn, Germany. Finally, we thank Pr. W. Thiemann for his support on the chemical analyses in Bremen, B. Pilette for his technical support on the SU5 beamline, C. Alcaraz for his major contribution to the polarimetry measurements, and A. Jones for his useful corrections.
Agarwal, V. K., Schutte, W., Greenberg, J. M., et al. 1985, Origins of Life, 16, 21

Bada, J. L. 1972, J. Am. Chem. Soc., 94, 1371

Bailey, J. 2001, Origins of Life and Evolution of the Biosphere, 31, 167

Bailey, J., Chrysostomou, A., Hough, J. H., et al. 1998, Science, 281, 672

Balavoine, G., Moradpour, A., \& Kagan, H. B. 1974, J. Am. Chem. Soc., 96, 5152

Bernstein, M. P., Sandford, S. A., Allamandola, L. J., Chang, S., \& Scharberg, M. A. 1995, ApJ, 454, 327

Bernstein, M. P., Dworkin, J. P., Sandford, S. A., Cooper, G. W., \& Allamandola, L. J. 2002, Nature, 416, 401

Bonner, W. A., \& Bean, B. D. 2000, Origins of Life and Evolution of the Biosphere, 30, 513

Brack, A., \& Spach, G. 1981, Origins of Life, 11, 135

Briggs, R., Ertem, G., Ferris, J. P., et al. 1992, Origins of Life and Evolution of the Biosphere, 22, 287

Buschermöhle, M., Whittet, D. C. B., Chrysostomou, A., et al. 2005, ApJ, 624, 821

Chyba, C. F., \& Sagan, C. 1992, Nature, 355, 125

Cronin, J. R., \& Pizzarello, S. 1997, Science, 275, 951

Cronin, J. R., \& Pizzarello, S. 1999, Adv. Space Res., 23, 293

Dartois, E., d'Hendecourt, L., Thi, W., Pontoppidan, K. M., \& van Dishoeck, E. F. 2002, A\&A, 394, 1057

Engel, M. H., \& Macko, S. A. 1997, Nature, 389, 265

Flores, J. J., Bonner, W. A., \& Massey, G. A. 1977, J. Am. Chem. Soc., 99, 3622

Gibb, E. L., Whittet, D. C. B., Boogert, A. C. A., \& Tielens, A. G. G. M. 2004, ApJS, 151, 35

Greenberg, J. M., \& Yencha, A. J. 1973, Interstellar Dust and Related Topics, IAU Symp., 52, 369

Hagen, W., Allamandola, L. J., \& Greenberg, J. M. 1979, Ap\&SS, 65, 215

d'Hendecourt, L. B., Allamandola, L. J., Baas, F., \& Greenberg, J. M. 1982, A\&A, 109, L12

d'Hendecourt, L. B., Allamandola, L. J., \& Greenberg, J. M. 1985, A\&A, 152, 130

Hough, J. H., Bailey, J. A., Chrysostomou, A., et al. 2001, Adv. Space Res., 27, 313

Huang, Z.-H., Wang, J., Gage, D. A., et al. 1993, J. Chromatogr., 635, 271

Jacobson, S. J., Willson, C. G., \& Rapoport, H. 1974, J. Org. Chem., 39, 1074

Jorissen, A., \& Cerf, C. 2002, Origins of Life and Evolution of the Biosphere, 32, 129

Manning, J. M. 1970, J. Am. Chem. Soc., 92, 7449

Meierhenrich, U. J., Muñoz Caro, G. M., Bredehöft, J.-H., Jessberger, E. K., \& Thiemann, W. H.-P. 2004, Proceedings of the National Academy of Science, 101,9182

Meierhenrich, U. J., Nahon, L., Alcaraz, C., et al. 2005, Angew. Chem. Int Ed., 44,5630

Muñoz Caro, G. M., \& Schutte, W. A. 2003, A\&A, 412, 121

Muñoz Caro, G. M., Meierhenrich, U. J., Schutte, W. A., et al. 2002, Nature, 416, 403

Muñoz Caro, G. M., Meierhenrich, U., Schutte, W. A., Thiemann, W. H.-P., \& Greenberg, J. M. 2004, A\&A, 413, 209

Nahon, L., \& Alcaraz, C. 2004, Appl. Opt., 43, 1024

Nahon, L., Corlier, M., Peaupardin, P., et al. 1997, Nucl. Instrum. Meth. A, 396, 237

Nahon, L., Alcaraz, C., Marlats, J.-L., et al. 2001, Rev. Sci. Instrum., 72, 1320

Nishino, H., Kosaka, A., Hembury, G. A., Matsushima, K., \& Inoue, Y. 2002, J. Chem. Soc., Perkin Trans. 2, 3, 582

Nuevo, M., Meierhenrich, U. J., d'Hendecourt, L., et al. 2005, Adv. Space Res., in press, available online

Oró, J. 1961, Nature, 190, 389

Pizzarello, S., \& Cronin, J. R. 2000, Geochim. Cosmochim. Acta, 64, 329

Pizzarello, S., Zolensky, M., \& Turk, K. A. 2003, Geochim. Cosmochim. Acta, 67,1589

Pizzarello, S., \& Weber, A. L. 2004, Science, 303, 1151

Pollock, G. E., Cronin, S. E., Kvenvolden, K. A., \& Cheng, C.-N. 1975, Geochim. Cosmochim. Acta, 39, 1571

Rubenstein, E., Bonner, W. A., Noyes, H, P., \& Brown, G. S. 1983, Nature, 306, 118

Shibata, T., Yamamoto, J., Matsumoto, N., et al. 1998, J. Am. Chem. Soc., 120, 12157

Snyder, P. A., Vipond, P. M., \& Johnson, W. C. Jr. 1973, Biopolymers, 12, 975

Wagner, E. L., \& Hornig, D. F. 1950, J. Chem. Phys., 18, 296

\section{References}

Abe, I., Fujimoto, N., Nishiyama, T., Terada, K., \& Nakahara, T. 1996, J. Chromatogr., 722, 221 